\title{
ANDES

www.scielo.cl

\section{Infancia y COVID-19: Los efectos indirectos de la pandemia COVID-19 en el bienestar de niños, niñas y adolescentes}

\author{
Childhood and COVID-19: The indirect effects of the COVID-19 pandemic \\ on the well-being of children and adolescents
}

\author{
Franco Díaz Rubio $^{\mathrm{a}, \mathrm{b}, \mathrm{c}, \mathrm{d}}$, Alejandro Donoso Fuentes ${ }^{\mathrm{a}, \mathrm{d}}$ \\ aHospital Clínico Metropolitano La Florida. Santiago, Chile \\ bHospital El Carmen de Maipú. Santiago, Chile \\ 'Escuela de Medicina, Universidad Finis Terrae. Santiago, Chile \\ dRed Colaborativa Pediátrica de Latinoamérica (LARed Network). Santiago, Chile
}

Recibido: 28 de enero de 2022; Aceptado: 1 de febrero de 2022

¿Qué se sabe del tema que trata este estudio?

La pandemia COVID-19 ha sido una crisis sanitaria, política y económica, que ha afectado en mayor medida a las poblaciones vulnerables. A diferencia de adultos y la tercera edad, la enfermedad grave por COVID-19 es infrecuente en niños, niñas y adolescentes (NNA). Sin embargo, ha ocurrido un gran deterioro del bienestar multidimensional de NNA durante los 2 años iniciales de la pandemia.

\begin{abstract}
¿Qué aporta este estudio a lo ya conocido?
Las medidas no farmacológicas de contención de la pandemia como el cierre de colegios, restricción de movilidad y confinamiento, resultaron en una desprotección de NNA y un retroceso en los programas de desarrollo y salud de la infancia. Para entender este fenómeno es necesario evaluar los factores más allá de los individuos: intrafamiliar (micronivel), instituciones de protección de la infancia (mesonivel), y la respuesta de la sociedad a la pandemia (macronivel). El tercer año de la pandemia es una oportunidad para centrarnos nuevamente en la salud y el bienestar de comunidades e individuos, y en forma urgente en el andamiaje construido para protección de NNA durante el último siglo.
\end{abstract}

\section{Resumen}

El impacto de la pandemia COVID-19 ha sido invisibilizado en niños, niñas y adolescentes, ya que muchos de los efectos negativos han sido producto de las medidas de contención y mitigación, y sólo serán cuantificables a mediano y largo plazo. Aunque la respuesta mundial ha sido exitosa en disminuir la letalidad de la enfermedad, el efecto nocivo en poblaciones vulnerables, como niños, niñas y adolescentes es de alto impacto, catalogado como catastrófico por organismos internacionales. La pandemia ha afectado profundamente la salud física y mental de este grupo etario, y sus efectos negativos silenciosos se extienden a diferentes ámbitos como la escolaridad, economía familiar, trabajo infantil y seguridad alimentaria. El tercer año de la pandemia es una oportunidad para incorporar el bienestar multidimensional de los niños, niñas y adolescentes como piedra angular de la respuesta de la sociedad a una crisis global, sea esta de salud, económica o política.
Palabras clave: COVID-19; Pandemia; Salud Infantil; Adolescencia; SARS-CoV-2 


\begin{abstract}
The impact of the COVID-19 pandemic has been overlooked in children and adolescents since many of the negative effects have been the result of containment and mitigation measures and will only be quantifiable in the medium and long term. Although the global response has been successful in reducing the lethality of the disease, the harmful effect on vulnerable populations, such as children and adolescents, is enormous and has been classified as catastrophic by international organizations. The pandemic has deeply affected the physical and mental health of children and adolescents, but also silently its negative effects extend across many areas such as schooling, familiar economy, child labor and food security. The third year of the pandemic is an opportunity to include the multidimensional well-being of children and adolescents as a cornerstone of society's response to a global crisis, whether health, economic or political.
\end{abstract}

Keywords: COVID-19; Pandemic; Child Health; Adolescents; SARS-CoV-2
Luego de transcurridos dos años de la pandemia por COVID-19, paulatinamente ha quedado en evidencia la desprotección sufrida por los niños, niñas y adolescentes (NNA) en diversos ámbitos de su salud física y psicológica. Durante el año 2020, mientras se intentaba contener la avalancha de casos y muertes a nivel global, la prensa, la sociedad civil, los gobiernos, investigadores y profesionales de la salud, se enfocaron principalmente en casos pediátricos excepcionales asociados a COVID-19 como el síndrome inflamatorio multisistémico (denominado MIS-C por el acrónimo en inglés de Multisystem inflammatory syndrome in children, o también conocido como PIMS por Pediatric Inflammatory Multisystem Syndrome), enfermedades autoinmunes relacionadas a COVID-19 y disminución de las infecciones respiratorias ${ }^{1-7}$. En este contexto, los efectos deletéreos directos e indirectos de la pandemia en NNA han sido inicialmente invisibilizados ${ }^{8,9}$. Los NNA, una población altamente vulnerable, están sufriendo las consecuencias de una enfermedad cuya incidencia, morbilidad y letalidad es excepcional en este grupo etario en ausencia de comorbilidades ${ }^{2,8-10}$, con una tasa de incidencia de COVID-19 ajustada en Chile, veinte veces menor que en la población general. Al igual que series internacionales, la incidencia en nuestro país de COVID-19 en NNA ha sido muy dependiente de la circulación comunitaria del virus, y no así de medidas específicas para limitar su exposición $^{11,12}$. Esto apoya a las observaciones que refieren que los niños en su mayoría se exponen al virus en su domicilio, y su rol en la transmisión y circulación viral a nivel poblacional es baja, a diferencia de otros virus, como la influenza ${ }^{13-16}$.

Los efectos indirectos de la pandemia son más difíciles de cuantificar y su instalación es lenta y silente $\mathrm{e}^{8,9,17,18}$. Si bien estos afectan a todos los individuos, los NNA presentan particularidades que deben ser evaluadas con detención para comprender la situación de urgencia en que nos encontramos. Los factores asociados a la pandemia que inciden en el bienestar de NNA pueden ser clasificados en tres niveles, micro, meso y macro, que van más allá del individuo. El nivel macro ha sido ampliamente revisado en la literatura internacional, no así los otros niveles asociados al estado de salud global de $\mathrm{NNA}^{17-20}$.

\section{Micro-nivel}

Comprende aquellos factores que ocurren a nivel familiar y su entorno. Las medidas de distanciamiento físico produjeron un aumento del tiempo de permanencia en el domicilio, muchas veces en espacios reducidos, cambiando en forma drástica la dinámica familiar. Importantes factores asociados han sido la inestabilidad económica por pérdida del trabajo del jefe de hogar, el surgimiento de trabajo informal, la pérdida de vivienda y hacinamiento producto de la presencia de diversos miembros de la familia concentradas en una sola residencia (hogares multigeneracionales), y fallecimiento de seres queridos. Estos factores pueden incidir en un deterioro de la salud mental de NNA, produciendo ansiedad, insomnio y síntomas depresivos, dependiendo de la capacidad de resiliencia personal y del grupo familiar. También estas condiciones hacen a los NNA más vulnerables a violencia intrafamiliar, traumatismos no intencionales e intencionales, abuso y otras experiencias biográficas adversas, cuyo efecto durará décadas y probablemente a escala transgeneracional, es decir en sus hijos y nietos ${ }^{17,20-26}$.

\section{Meso-nivel}

Estos factores están asociados a las estructuras y organizaciones que protegen a los NNA, como el sistema de salud y educacional. La respuesta gerontocéntrica de la salud pública contribuyó inevitablemente al abandono de los NNA. La instalación de las medidas no-farmacológicas destinadas a la contención del virus 
produjeron aislamiento social, sedentarismo, inseguridad alimentaria, influyendo directamente en el bienestar y desarrollo de una vida saludable $8,9,17,27$.

El aumento de recursos monetarios, humanos y tecnológicos para el manejo de casos de COVID-19 en adultos con falla respiratoria en la red integrada público/privada, estuvo acompañada de cierre de servicios de urgencia pediátricos, centros ambulatorios de pediatría general, especialidades y Unidades de Cuidados Intensivos ${ }^{28}$. Afortunadamente, las medidas de distanciamiento físico produjeron una gran caída de las enfermedades infecciosas infantiles, disminuyendo las consultas en los servicios de urgencia y las hospitalizaciones pediátricas ${ }^{1,3,4,28}$. En paralelo, los NNA con enfermedades crónicas tuvieron un pobre control con profesionales de la salud, como pacientes con diabetes mellitus tipo I y epilepsia, produciendo descompensaciones por falta de supervisión y disponibilidad oportuna de medicamentos. Otro efecto negativo fue la inevitable merma en el diagnóstico precoz de enfermedades no transmisibles, por ejemplo, cáncer infantil, el retraso en la resolución adecuada de patologías quirúrgicas y en consultas pertinentes en la atención primaria de salud y de especialidades, particularmente en grupos de niños altamente vulnerables. Todas estas consecuencias, producto de la magnitud y duración de las restricciones impuestas, fueron graves y prolongadas en la mayoría de los países latinoamericanos ${ }^{29-36}$.

El cierre de colegios fue una de las medidas precozmente adoptadas a nivel mundial, respaldada casi unánimemente por padres, profesores, población general, especialistas, prensa y los gobiernos. La experiencia previa con la pandemia por virus influenza (H1N1) apoyaba la instauración de esta medida extrema en forma empírica, asumiendo los profundos efectos negativos en NNA, más allá de la perdida educacional, sino ámbitos tan diversos como la seguridad alimenta$\operatorname{ria}^{37,38}$. La evidencia surgida tempranamente durante la pandemia logró determinar, aún antes del inicio de la vacunación masiva, que el efecto del cierre de colegios en la circulación comunitaria y muertes era marginal, los brotes ocurridos en colegios fueron excepcionales, $\mathrm{y}$ que los contagios de los NNA ocurrían habitualmente por contacto con adultos infectados, especialmente en localización intra-domiciliaria ${ }^{39-41}$. Sorprendentemente, luego de recabada esta información, se mantuvieron los cierres de colegios, asumiendo los profundos efectos negativos en esta población por una sociedad que pedía endurecimientos de medidas, como cuarentena total, para intentar frenar el avance de casos y muertes por COVID-19 $9^{42,43}$. Se estima que 160 millones de NNA sólo en Latinoamérica tuvo una interrupción de la continuidad educativa ${ }^{37}$. En Chile durante el primer año de la pandemia el mandato de cierre de los centros educacionales fue completo, afectando a más de 3 mi- llones de niños, un $61 \%$ en enseñanza básica y un 12\% en pre-escolar ${ }^{44}$. Las medidas inicialmente transitorias como la modalidad a distancia o en línea, rápidamente adquirieron un carácter permanente. El éxito de las actividades educativas a distancia depende de múltiples factores. La magnitud del menoscabo del desarrollo y aprendizaje es inversamente proporcional a la edad de los estudiantes, siendo pre-escolares, seguidos de niños en educación básica, los más damnificados de la nopresencialidad en centros de educación.

Chile es uno de los países con mayor conectividad y acceso a internet de la región. Sin embargo, las condiciones para educación a distancia son dramáticamente disímiles. Al comienzo de la pandemia menos de un tercio de los profesores tenían preparación formal en educación a distancia, concentrándose principalmente en la educación privada. Concordantemente, los implementos tecnológicos disponibles son limitados en colegios públicos y particulares con subvención estatal, que corresponden a casi el $90 \%$ de la matrícula. Las condiciones para una experiencia educativa fueron inadecuadas en la mayoría de los $\operatorname{casos}^{45}$. Por ejemplo, más del 50\% de los NNA residen en viviendas de menos de $50 \mathrm{~m}^{2}$, sin un espacio propio para realizar adecuadamente sus actividades y la mayoría de ellos considera una calidad de internet insuficiente para las actividades asignadas. En los NNA que asisten a escuelas privadas, correspondiente al $10 \%$ de la matrícula, la situación es diametralmente opuesta. La mayoría reside en viviendas mayores a $100 \mathrm{~m}^{2}$, con un espacio y dispositivo exclusivo para las actividades educativas ${ }^{45}$.

Finalmente, todo esto se resume en que la cantidad de horas lectivas y el tiempo dedicado para actividades remotas (tareas, conexión en línea, entre otras) puede tener una diferencia hasta cinco veces, lo que en suma produce una divergencia exponencial e irrecuperable, siendo los grupos vulnerables los grandes damnifica$\operatorname{dos}^{45-48}$.

En Chile, la programación regreso a clases presenciales durante 2021 también resultó en una sorprendente disparidad. Estudiantes de colegios sin subvención rápidamente retomaron clases semi-presenciales y presenciales, gracias a su infraestructura basal, inversión en sistemas y protocolos de seguridad sanitaria. Mientras que menos del $5 \%$ de colegios públicos reanudó las clases en forma regular debido a una estructura basal deficiente, falta de recursos destinados a la mejoría de su infraestructura y las particularidades socioculturales de padres, funcionarios, administradores, líderes de opinión y políticos. Un ejemplo tangible de esto fue el requerimiento de vacunación a funcionarios y NNA para cerciorar un "aula segura". Los datos internacionales también dan cuenta de esta heterogeneidad y el aumento de la brecha educativa ocasionada por la pandemia ${ }^{9,43,47-50}$. 


\section{Macro-nivel}

Este nivel corresponde a la respuesta de la sociedad y los gobiernos al COVID-19. Las medidas no farmacológicas de control de la pandemia han producido un estrés crónico en las comunidades locales, nacionales e internacionales ${ }^{51}$. A diferencia de acontecimientos como desastres naturales y atentados terroristas masivos, la magnitud global y la exposición permanente a información relacionada a la pandemia como son la diseminación viral, cifras de mortalidad y desarrollo de nuevas cepas, tanto en la prensa como en redes sociales, no han permitido afrontarla de una forma adecuada. Esto ha afectado especialmente a los adolescentes, cuyo principal acceso de información son las redes sociales y prensa digital. La desinformación, noticias falsas (traducción del termino anglosajón, fake-news) y la información por parte de no expertos han originado inevitablemente una pérdida de la confianza en las instituciones establecidas y de referencia tradicionales. Una característica de los adolescentes en las redes sociales es la generación cámaras de eco (traducción del termino anglosajón, echo-chamber) ${ }^{52}$. Este fenómeno se refiere a cómo a partir de unas cuantas afirmaciones iniciales, la información se disemina rápidamente por individuos y grupos con ideas afines, retornando como retroalimentación de verdad absoluta a los mismos individuos, independiente la veracidad, racionalidad o fuente de origen. Este fenómeno produce un sesgo de confirmación y una perspectiva absoluta y cerrada, similar al fenómeno de visión en túnel. En forma asociada, también se forman burbujas epistémicas, que se refiere al cese de exposición a ideas y explicaciones discordantes o contrarias. Frecuentemente los tópicos de desinformación incluyen a NNA, como, por ejemplo, señalar a los niños como "súper propagadores", el riesgo de retención de dióxido de carbono por mascarillas, el ser responsabilizados de contagios intradomiciliarios $^{19}$, las presencia de microchips en vacunas o que su finalidad era enfermar a la población general. Los adolescentes están expuestos en forma directa a noticias falsas, siendo altamente vulnerables y propicios a continuar su diseminación ${ }^{53,54}$.

Contrario al sentido común, el inicio de los exitosos programas de vacunación no mejoró la sensación de seguridad de NNA en su lugar de residencia. Al ver la persistencia elevada de casos a pesar de la amplia vacunación de la población objetivo, rápidamente muchos sectores de la sociedad pidieron la vacunación de NNA para disminuir la circulación comunitaria del virus, por ende, ser responsables de la segunda ola. Es así como en Chile a sólo seis meses de iniciada la vacunación contra COVID-19, comenzó el uso de la vacuna mRNA BNT162b2 en adolescentes entre 12 y 17 años (22 de junio 2021). En los seis meses siguientes, mien- tras se superaba la vacunación del $80 \%$ de la población objetivo y se comenzaba a administrar las primeras dosis de refuerzo en adultos, se obtuvo aprobación de emergencia para vacunar a niños entre 3 y 11 años con CoronaVac ${ }^{\oplus}$ (Sinovac, BioTech, China). Muchos países de la región siguieron estas iniciativas, aunque esta conducta no ha sido homogénea sobre el tipo de vacuna, edad de administración y obligatoriedad, lo que refleja las discrepancias técnicas y politicas mundiales de salud pública respecto a NNA y COVID-19. A modo de ejemplo, el Reino Unido no ha aprobado el uso de vacunas en menores de 12 años, mientras que Estados Unidos aprobó la vacuna Pfizer-BioNTech para mayores de 5 años. La vacunación de adolescentes es efectiva en reducir COVID-19 grave en ese grupo, pero el costo beneficio para NNA y su efecto en el control de brotes o sobre el total de casos comunitarios es aún tema de debate entre expertos, pero aparentemente es muy bajo ${ }^{55-61}$. Sin embargo, contrario a estos datos incipientes, el 01 de noviembre 2021 Chile implementó un pasaporte sanitario como prueba de vacunación para menores de edad ${ }^{62}$. De este modo, Chile es unos de los pocos países que solicitan este documento para que menores realicen ciertas actividades, dependiendo de las restricciones impuestas a la población general ${ }^{62}$. Siendo una medida muy controversial, es llamativa la ausencia de un debate con una participación amplia de la sociedad, y no exclusivamente epidemiológico/ infectológico. Este abandono de la discusión constructiva ha ocurrido también en otros países ${ }^{55,56,63-65}$.

Un ejemplo tangible sobre las medidas tomadas que incluyen a NNA, pero sin evaluar sus consecuencias, fue el reporte inicial de miocarditis en adolescentes (y hombres jóvenes) asociada a vacunas mRNA ${ }^{66-69}$. Hubo reticencia de entes reguladores, sociedades académicas y publicaciones científicas, que prefirieron caer en el descrédito asociándolo con movimientos antivacunas y libertarios ${ }^{70,71}$. Se minimizó esta tenue señal de alarma inicial, aún considerando que la vacuna tenía una aprobación de emergencia, es decir, un producto farmacéutico que en situaciones habituales (no de pandemia) no había sido aprobada para uso en menores de edad, especialmente si es un grupo etario con un riesgo bajo de morbimortalidad y el beneficio de salud pública no es claro. Finalmente, luego de millones de dosis administradas, el análisis de datos mostró un mayor riesgo miocarditis en hombres entre 12 y 17 años, en su mayoría de carácter leve, especialmente luego de la administración de la segunda dosis o refuerzos ${ }^{72-74}$. La posición adoptada por entes reguladores y referentes mundiales, como los Centros de Control y Prevención de Enfermedades (CDC, del inglés, Centers for Disease Control and Prevention) de los Estados Unidos y la Agencia Federal de Drogas (FDA, del inglés, Federal Drug Agency) de Norteamérica, no han incorporado 
estos datos esenciales. A diferencia de lo que muchos creen, estas observaciones no intentan evitar vacunaciones, sino más bien reevaluar el tipo de vacuna administrada en adolescentes, su frecuencia, y alertar a médicos sobre los eventuales síntomas. La falta de una estrategia proactiva de dichas organizaciones dio cabida a la entrega de información a la población general a través de la prensa y otros medios de difusión, un error que desencadenó una ola de comentarios, a veces llevados al extremo, en redes sociales ${ }^{53,75-81}$. Se ha propuesto que otro efecto beneficioso de vacunar a NNA contra COVID-19 pudiera ser la prevención síndrome de COVID-19 prolongado o síndrome de COVID-19. Este cuadro clínico es aún pobremente definido y no hay consenso entre los expertos. Incluye cualquier síntoma que permanezca más de 4 semanas después de la infección. Con los datos disponibles, aparentemente su frecuencia es mucho más baja de lo que inicialmente se pensó, pero es un área de investigación en la actualidad. ${ }^{81,84}$

El desconocimiento del nuevo agente infeccioso, su rapidez de propagación, extensión geográfica y gravedad, produjeron razonable e ineludiblemente un constante cambio de las propuestas de expertos y las medidas adoptadas por los gobiernos. Si bien el primer año de la pandemia era esperable la incertidumbre, la improvisación y los errores, en el segundo año las medidas impulsadas férreamente en un comienzo debieran haber sido evaluadas, modificando y enfocando las estrategias, educando a la población y asumiendo y corrigiendo las políticas desacertadas, que en el tiempo se mostraron fútiles. La Figura 1 muestra indicadores seleccionados de restricciones en Chile durante la pandemia.

Luego de dos años de pandemia, es necesario una mirada a mediano y largo plazo, enfocándose en los NNA para esta nueva etapa de pandemia. La UNICEF ha catalogado la situación de la infancia durante la pandemia como un desastre, cuyos efectos a largo plazo son devastadores, retrocediendo casi diez años en los objetivos de desarrollo del milenio de las Naciones Unidas $^{18,33}$.

Toda muerte inesperada y prematura conlleva una carga emocional en familiares y las comunidades, especialmente cuando son evidenciados como exceso de mortalidad en la sociedad. Afortunadamente, muchos especialistas lentamente han reflexionado del tema, logrando cruzar el abismo impuesto por la tanatofobia tan característica de las sociedades occidentales y los números inertes de modelos predictivos, para centrarse nuevamente en la salud y el bienestar de comunidades e individuos a mediano y largo plazo. Los pediatras y todos los profesionales de la salud, estamos llamados con urgencia a promover y retomar el andamiaje construido durante el último siglo para el bienestar de NNA. Las medidas iniciales parecen ser bastante simples, pero lo dificultoso es que debe participar la sociedad en su conjunto. Por ejemplo:

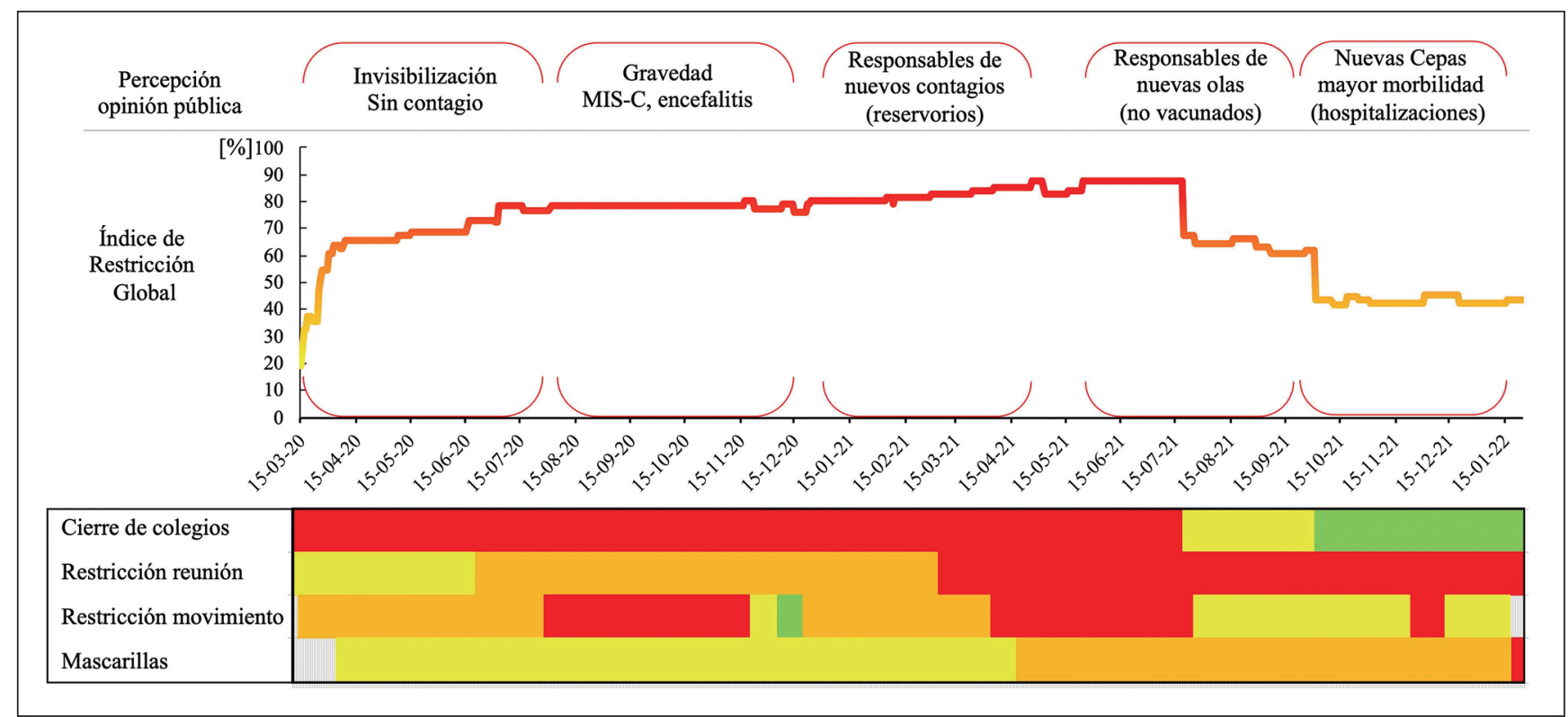

Figura 1. Elementos seleccionados de restricciones en Chile desde 15.03.20 a 15.01.22. Gradiente de colores representa dureza de restricciones, verde sin restricción, rojo alta restricción,situación más restrictiva en colores oscuros. El índice de restricción global es un indicador que integra múltiples elementos, y se expresa en forma porcentual desde 0\%, sin restricciones, a 100\%, máximo de restricciones. Datos obtenidos de Oxford COVID-19 Government Response Tracker, open dataset identifier: oxford-covid-19-government-response-tracker ${ }^{92}$. 
1) recuperar los colegios e instituciones de enseñanza, como un lugar multidimensional de cuidado de NNA, que incluye, salud, seguridad alimentaria, e integración social;

2) promover los programas exitosos en la atención primaria, hasta la atención de subespecialidades, como control de la díada madre-hijo, inmunizaciones de enfermedades transmisibles, diagnóstico y tratamiento precoz de enfermedades oncológicas, y acondicionar en forma prioritaria para los NNA lugares educacionales y de salud, incluyendo prevención;

3) abordar con especial urgencia los problemas agudos de NNA, como es salud mental, enfermedades transmisibles prevenibles y el deterioro del estado nutricional;

4) incorporar, adicionalmente, el efecto de medidas sanitarias para nuevas olas de COVID-19 en NNA, considerando más allá de la morbilidad y mortalidad aguda, como, por ejemplo, con métricas conocidas como los años de vida potencialmente $\operatorname{perdidos}^{85}$;

5) participar activamente en la educación de cultura médica, también denominada alfabetización científica de la sociedad (en inglés science literacy) ${ }^{86-88}$;

6) convocar en forma urgente a la conversación pendiente entre los gobiernos, investigadores, entes regulatorios y representantes diversos de la sociedad, para afrontar los próximos años de pandemia y post-pandemia ${ }^{89,90}$.
Los NNA en todo el mundo están sufriendo silenciosamente consecuencias de la pandemia por COVID-19, muchas de ellas producidas por las respuestas de contención y mitigación de ellas. Estas medidas extraordinarias, que innegablemente han sido exitosas en disminuir la letalidad de la enfermedad, incluyen el distanciamiento físico, compra de insumos y respuesta hospitalaria junto con una coordinación y cooperación internacional sin precedentes entre gobiernos, entes reguladores, industria farmacéutica, científicos e inversores, que permitieron el rápido desarrollo de vacunas con tecnología de punta. En forma similar, es posible esperar una cooperación multipartita de similar magnitud para enfocarnos en el bienestar multidimensional de NNA. Sin embargo, para avanzar lo primero es lograr salir del espectro de la coronafobia y el coronacentrismo, para no repetir los mismos errores del comienzo de la pandemia. Este tercer año de pandemia es la oportunidad para enmendar el rumbo, para cuando a futuro los coronials y cuarenteens cuestionen los efectos de los hechos del comienzo de esta década. ${ }^{91}$

\section{Conflicto de intereses}

Los autores declaran no tener conflicto de intereses.

\section{Referencias}

1. Vásquez-Hoyos P, Diaz-Rubio F, Monteverde-Fernandez N, et al. Reduced PICU respiratory admissions during COVID-19. Arch Dis Child. 2021; 106:808-11. doi:10.1136/ archdischild-2020-320469

2. Zimmermann P, Curtis N. Coronavirus Infections in Children Including COVID-19: An Overview of the Epidemiology, Clinical Features, Diagnosis, Treatment and Prevention Options in Children. Pediatr Infect Dis J. 2020; 39:355-68. doi: 10.1097/ INF.0000000000002660

3. Diesner-Treiber SC, Voitl P, Voitl JJM, et al. Respiratory Infections in Children During a Covid-19 Pandemic Winter. Front Pediatr. 2021; 9:740785. doi:10.3389/ fped.2021.740785

4. Friedrich F, Ongaratto R, Scotta MC, et al. Early Impact of Social Distancing in Response to Coronavirus Disease 2019 on Hospitalizations for Acute Bronchiolitis in Infants in Brazil. Clin Infect Dis. 2021;
72(12):2071-2075. doi:10.1093/cid/ ciaa 1458

5. Davies P, Evans C, Kanthimathinathan $\mathrm{HK}$, et al. Intensive care admissions of children with paediatric inflammatory multisystem syndrome temporally associated with SARS-CoV-2 (PIMS-TS) in the UK: a multicentre observational study [published correction appears in Lancet Child Adolesc Health. 2020 Jul 17]. Lancet Child Adolesc Health. 2020;4(9):669-677. doi:10.1016/S23524642(20)30215-7

6. Yonker LM, Neilan AM, Bartsch Y, et al. Pediatric Severe Acute Respiratory Syndrome Coronavirus 2 (SARS-CoV-2): Clinical Presentation, Infectivity, and Immune Responses. J Pediatr. 2020;227:45-52.e5. doi:10.1016/j. jpeds.2020.08.037

7. González-Dambrauskas S, VásquezHoyos P, Camporesi A, et al. Pediatric Critical Care and COVID-19. Pediatrics. 2020;146(3):e20201766. doi:10.1542/ peds.2020-1766.

8. Kadambari S, Goldacre R, Morris E, Goldacre MJ, Pollard AJ. Indirect effects of the covid-19 pandemic on childhood infection in England: population based observational study. BMJ. 2022;376:e67519. doi:10.1136/bmj-2021067519

9. Camporesi A, Díaz-Rubio F, Carroll CL, González-Dambrauskas S. Protecting children from iatrogenic harm during COVID19 pandemic. J Paediatr Child Health. 2020;56(7):1010-1012. doi:10.1111/jpc.14989

10. INFORME EPIDEMIOLÓGICO DE NIÑOS, NIÑAS Y ADOLESCENTES CON COVID-19 CHILE, Semana epidmeiológica 9 año 2020 a la 36 año 2021. Departamento de Epidemiologia, Ministerio de Salud, Chile. Disponible en: <http://epi.minsal.cl/wp-content/ uploads/2021/09/Informe-epidemCOVID-19_NNA_SE36_Final.pdf $>$. Accedido 10.01.22

11. INFORME EPIDEMIOLÓGICO No167. ENFERMEDAD POR SARS-CoV-2 (COVID-19) CHILE. 3 de enero de 2022. Departamento de Epidemiologia, Ministerio de Salud, Chile. Disponible en: <https://www.minsal.cl/wp- 
content/uploads/2022/01/InformeEpidemiol\%C3\%B3gico-N\%C2\%B0167. pdf $>$. Accedido 10.01.22

12. Mensah AA, Sinnathamby M, Zaidi A, et al. SARS-CoV-2 infections in children following the full re-opening of schools and the impact of national lockdown: Prospective, national observational cohort surveillance, July-December 2020, England. J Infect. 2021;82(4):67-74. doi:10.1016/j.jinf.2021.02.022

13. Gaythorpe KAM, Bhatia S, Mangal T, Unwin HJT, Imai N, Cuomo-Dannenburg $\mathrm{G}$, et al. Children's role in the COVID-19 pandemic: a systematic review of early surveillance data on susceptibility, severity, and transmissibility. Sci Rep-uk. 2021;11:13903. doi:10.1038/s41598-02192500-9

14. Imamura $\mathrm{T}$, Saito $\mathrm{M}$, Ko $\mathrm{YK}$, et al. Roles of Children and Adolescents in COVID-19 Transmission in the Community: A Retrospective Analysis of Nationwide Data in Japan. Front Pediatr. 2021;9:705882. doi:10.3389/ fped.2021.705882

15. Munro APS, Faust SN. Children are not COVID-19 super spreaders: time to go back to school [published correction appears in Arch Dis Child. 2021;106(2):e9]. Arch Dis Child. 2020;105(7):618-619. doi:10.1136/ archdischild-2020-319474

16. Viner R, Waddington C, Mytton O, et al. Transmission of SARS-CoV-2 by children and young people in households and schools: A meta-analysis of populationbased and contact-tracing studies. J Infect. 2021;S0163-4453(21)00633-2. doi:10.1016/j.jinf.2021.12.026

17. Faust SN, Munro APS. It's Time to Put Children and Young People First During the Global COVID-19 Pandemic. JAMA Pediatr. 2021;175(2):127-128. doi:10.1001/jamapediatrics.2020.4582

18. Alkire S, Nogales R, Quinn NN, Suppa N. Global multidimensional poverty and COVID-19: A decade of progress at risk?. Soc Sci Med. 2021;291:114457. doi:10.1016/j.socscimed.2021.114457

19. Cevallos-Robalino D, Reyes-Morales N, Rubio-Neira M. Evolución e impacto de la infodemia en la población infantil en tiempos de COVID-19. Revista Panam De Salud Pública. 2021;45:e38.

20. Singh A, Shah N, Mbeledogu C, Garstang J. Child wellbeing in the United Kingdom following the COVID-19 lockdowns. Paediatr Child Health (Oxford). 2021;31(12):445-448. doi:10.1016/j. paed.2021.09.004

21. Racine N, McArthur BA, Cooke JE, Eirich R, Zhu J, Madigan S. Global Prevalence of Depressive and Anxiety Symptoms in Children and Adolescents During COVID-19: A Meta-analysis.
JAMA Pediatr. 2021;175(11):1142-1150. doi:10.1001/jamapediatrics.2021.2482

22. Brown SM, Doom JR, Lechuga-Peña S, Watamura SE, Koppels T. Stress and parenting during the global COVID-19 pandemic. Child Abuse Negl. 2020;110 (Pt 2):104699. doi:10.1016/j. chiabu.2020.104699.

23. Bignardi G, Dalmaijer ES, Anwyl-Irvine $\mathrm{AL}$, et al. Longitudinal increases in childhood depression symptoms during the COVID-19 lockdown. Arch Dis Child. 2020;106(8):791-797. doi:10.1136/ archdischild-2020-320372

24. Kovler ML, Ziegfeld S, Ryan LM, et al. Increased proportion of physical child abuse injuries at a level I pediatric trauma center during the Covid-19 pandemic. Child Abuse Negl. 2021;116(Pt 2):104756. doi:10.1016/j.chiabu.2020.104756

25. Hashmi PM, Zahid M, Ali A, et al. Change in the spectrum of orthopedic trauma: Effects of COVID-19 pandemic in a developing nation during the upsurge; a cross-sectional study. Ann Med Surg (Lond). 2020;60:504-508. doi:10.1016/j. amsu.2020.11.044

26. Solís F. F, Saavedra O. R, Zalavari P. K, Paredes M. C. Efectos del confinamiento por COVID-19 en la demanda de atención de niñas, niños y adolescentes con quemaduras. Andes pediatr. 2021;92(6): 854-861. doi:10.32641/ andespediatr.v92i6.3620.

27. Fuentes-Barría H, Aguilera-Eguía R, González-Wong C. Inseguridad alimentaria y obesidad: una mirada más allá del sedentarismo y malnutrición en la pandemia COVID-19. Andes pediatr. 2021;92(5): 807-808. doi:10.32641/ andespediatr.v92i5.3933.

28. Díaz F, Kehr J, Cores C, et al. Clinical and organizational framework of repurposing pediatric intensive care unit to adult critical care in a resource-limited setting: Lessons from the response of an urban general hospital to the COVID-19 pandemic. J Crit Care. 2021;68:59-65. doi:10.1016/j.jcrc.2021.12.004

29. Hashmi PM, Zahid M, Ali A, et al. Change in the spectrum of orthopedic trauma: Effects of COVID-19 pandemic in a developing nation during the upsurge; a cross-sectional study. Ann Med Surg (Lond). 2020;60:504-508. doi:10.1016/j. amsu.2020.11.044

30. Lazzerini M, Barbi E, Apicella A, Marchetti F, Cardinale F, Trobia G. Delayed access or provision of care in Italy resulting from fear of COVID-19. Lancet Child Adolesc Health. 2020;4(5):e10-e11. doi:10.1016/S2352-4642(20)30108-5

31. Shuffrey LC, Firestein MR, Kyle MH, et al. Association of Birth During the COVID-19 Pandemic With Neurodevelopmental Status at 6 Months in Infants With and Without In Utero Exposure to Maternal SARS-CoV-2 Infection. JAMA Pediatr. 2022;e215563. doi:10.1001/ jamapediatrics.2021.5563

32. Wood ME, Delgado M, Jonsson Funk M. Understanding the Effects of the Pandemic on Infant DevelopmentThe Preterm Problem. JAMA Pediatr. 2022;e215570. doi:10.1001/ jamapediatrics.2021.5570.

33. COVID-19 pandemic leads to major backsliding on childhood vaccinations, new WHO, UNICEF data shows. Disponible en: $<\mathrm{https} / / / \mathrm{www}$.unicef. org/press-releases/covid-19-pandemicleads-major-backsliding-childhoodvaccinations-new-who-unicef-data $>$. Accedido 15.01.22.

34. DeSilva MB, Haapala J, Vazquez-Benitez G, et al. Association of the COVID-19 Pandemic With Routine Childhood Vaccination Rates and Proportion Up to Date With Vaccinations Across 8 US Health Systems in the Vaccine Safety Datalink. JAMA Pediatr. 2022;176(1):6877. doi:10.1001/jamapediatrics.2021.4251

35. DiMeglio LA. COVID-19 and Type 1 Diabetes: Addressing Concerns and Maintaining Control. Diabetes Care. 2021;44(9):1924-1928. doi:10.2337/dci210002

36. Conlon C, McDonnell T, Barrett M, et al. The impact of the COVID-19 pandemic on child health and the provision of Care in Paediatric Emergency Departments: a qualitative study of frontline emergency care staff. BMC Health Serv Res. 2021;21(1):279. doi:10.1186/s12913-02106284-9

37. Higgins CD, Páez A, Kim G, Wang J. Changes in accessibility to emergency and community food services during COVID-19 and implications for low income populations in Hamilton, Ontario. Soc Sci Med. 2021;291:114442. doi:10.1016/j.socscimed.2021.114442

38. Adams EL, Caccavale LJ, Smith D, Bean MK. Food Insecurity, the Home Food Environment, and Parent Feeding Practices in the Era of COVID-19. Obesity (Silver Spring). 2020;28(11):2056-2063. doi:10.1002/oby.22996

39. Ismail SA, Saliba V, Lopez Bernal J, Ramsay ME, Ladhani SN. SARS-CoV-2 infection and transmission in educational settings: a prospective, cross-sectional analysis of infection clusters and outbreaks in England. Lancet Infect Dis. 2021;21(3):344-353. doi:10.1016/S14733099(20)30882-3

40. Nunziata F, Poeta M, Vassallo E, et al. No Spread of SARS-CoV-2 From Infected Symptomatic Children to Parents: A Prospective Cohort Study in a Controlled Hospital Setting. Front 
Pediatr. 2021;9:720084. doi:10.3389/ fped.2021.720084

41. Calvani M, Cantiello G, Cavani M, et al. Reasons for SARS-CoV-2 infection in children and their role in the transmission of infection according to age: a case-control study. Ital J Pediatr. 2021;47(1):193. doi:10.1186/s13052-02101141-1

42. Tan W. School closures were overweighted against the mitigation of COVID-19 transmission: A literature review on the impact of school closures in the United States. Medicine (Baltimore). 2021;100(30):e26709. doi:10.1097/ MD.0000000000026709

43. Buonsenso D, Roland D, De Rose C, et al. Schools Closures During the COVID-19 Pandemic: A Catastrophic Global Situation. Pediatr Infect Dis J. 2021;40(4):e146-e150. doi:10.1097/ INF.0000000000003052

44. Niños, niñas y adolescentes en Chile 2020. Disponible en: <https://www.unicef.org/ chile/media/3371/file/Infancia\%20en $\% 20$ cifras.pdf $>$. Accedido 12.11.22.

45. Ponce T, Bellei C, VIelma C. Experiencias educativas en casa de niñas y niños durante la pandemia. Centro de Investigación Avanzada en Educación, Universidad de Chile. Disponible en: $<$ https://www.uchile.cl/documentos/ primer-informe-de-resultados-encuestaexperiencias-educativas-en-casa-deninas-y-ninos-durante-la-pandemia-pdf598-kb_172051_0_0454.pdf >. Accedido 12.11.22.

46. Hanno EC, Fritz LS, Jones SM, Lesaux NK. School Learning Format and Children's Behavioral Health During the COVID-19 Pandemic. JAMA Pediatr. 2022;10.1001/jamapediatrics.2021.5698. doi:10.1001/jamapediatrics.2021.5698

47. La educación en tiempo de la pandemia. Informe COVID-19 CEPAL-UNESCO. Disponible en: <https://repositorio.cepal. org/bitstream/handle/11362/45904/1/ S2000510_es.pdf $>$. Accedido 12.01.22.

48. van de Werfhorst HG. Inequality in learning is a major concern after school closures. Proc Natl Acad Sci U S A. 2021;118(20):e2105243118. doi:10.1073/ pnas. 2105243118

49. Goudeau S, Sanrey C, Stanczak A, Manstead A, Darnon C. Why lockdown and distance learning during the COVID-19 pandemic are likely to increase the social class achievement gap. Nat Hum Behav. 2021;5(10):1273-1281. doi:10.1038/s41562-021-01212-7

50. Limbers CA. Factors Associated with Caregiver Preferences for Children's Return to School during the COVID-19 Pandemic. J Sch Health. 2021;91(1):3-8. doi:10.1111/josh.12971

51. Taylor S, Landry CA, Paluszek MM,
Fergus TA, McKay D, Asmundson GJG. COVID stress syndrome: Concept, structure, and correlates. Depress Anxiety. 2020;37(8):706-714. doi:10.1002/da.23071

52. Cinelli M, De Francisci Morales G, Galeazzi A, Quattrociocchi W, Starnini M. The echo chamber effect on social media. Proc Natl Acad Sci U S A. 2021;118(9):e2023301118. doi:10.1073/ pnas. 2023301118

53. Pilgrim J, Vasinda S. Fake News and the "Wild Wide Web": A Study of Elementary Students' Reliability Reasoning. Societies. 2021; 11(4):121. doi: 10.3390/soc1104012

54. Herrero-Diz P, Conde-Jiménez J, Cózar SR de. Teens' Motivations to Spread Fake News on WhatsApp. Soc Media Soc. 2020; 6:2056305120942879. doi: $10.1177 / 2056305120942879$

55. Ali K, Berman G, Zhou H, et al. Evaluation of mRNA-1273 SARS-CoV-2 Vaccine in Adolescents. N Engl J Med. 2021;385(24):2241-2251. doi:10.1056/ NEJMoa2109522

56. Zimmermann P, Pittet LF, Finn A, Pollard AJ, Curtis N. Should children be vaccinated against COVID-19? Arch Dis Child. 2021; archdischild-2021-323040. doi:10.1136/archdischild-2021-323040

57. deSante-Bertkau JE, Knilans TK, Persad G, Zettler PJ, Fernandez Lynch H, Matheny Antommaria AH. Off-Label Prescription of COVID-19 Vaccines in Children: Clinical, Ethical, and Legal Issues. Pediatrics. 2021; e2021054578. doi:10.1542/peds.2021-054578

58. Wilhelm-Bavestrello J. Inmunizar a nuestros niños contra COVID-19 [Vaccinating our children against COVID-19]. Andes Pediatr. 2021;92(4):499-500. doi:10.32641/ andespediatr.v92i4.3878

59. Havers FP, Whitaker M, Self JL, Chai SJ, Kirley PD, Alden NB, et al. Hospitalization of Adolescents Aged 12-17 Years with Laboratory-Confirmed COVID-19 - COVID-NET, 14 States, March 1, 2020-April 24, 2021. Morbidity Mortal Wkly Rep. 2021;70:851-7.

60. Opel DJ, Diekema DS, Ross LF. Should We Mandate a COVID-19 Vaccine for Children?. JAMA Pediatr. 2021;175(2):125-126.

doi:10.1001/jamapediatrics.2020.3019

61. Plotkin SA, Levy O. Considering Mandatory Vaccination of Children for COVID-19. Pediatrics. 2021;147(6):e2021050531. doi:10.1542/ peds.2021-050531

62. Actualización plan "Paso a Paso", Gobierno de Chile. Disponible en: $<$ https://www.gob.cl/coronavirus/ pasoapaso/ > . Accedido 14.11.22

63. González-Dambrauskas S. El discriminar a un niño por su estado vacunal para covid19 es violar sus derechos. Disponible en: <https://link.medium.com/ gx65iZjT5mb $>$. Accedido 20.01.22.

64. Prasad V. At a time when the U.S. needed Covid-19 dialogue between scientists, Francis Collins moved to shut it down. Disponible en <https://www.statnews. com/2021/12/23/at-a-time-when-the-us-needed-covid-19-dialogue-betweenscientists-francis-collins-moved-to-shutit-down/>. Accedido 10.01.22.

65. Kofman A, Kantor R, Adashi EY. Potential COVID-19 Endgame Scenarios: Eradication, Elimination, Cohabitation, or Conflagration?. JAMA. 2021;326(4):303-304. doi:10.1001/ jama.2021.11042

66. Marshall M, Ferguson ID, Lewis P, et al. Symptomatic Acute Myocarditis in 7 Adolescents After Pfizer-BioNTech COVID-19 Vaccination. Pediatrics. 2021;148(3):e2021052478. doi:10.1542/ peds.2021-052478

67. Das BB, Kohli U, Ramachandran P, et al. Myopericarditis after messenger RNA Coronavirus Disease 2019 Vaccination in Adolescents 12 to 18 Years of Age. J Pediatr. 2021;238:26-32.e1. doi:10.1016/j. jpeds.2021.07.044

68. Dionne A, Sperotto F, Chamberlain S, et al. Association of Myocarditis With BNT162b2 Messenger RNA COVID-19 Vaccine in a Case Series of Children. JAMA Cardiol. 2021;6(12):1446-1450. doi:10.1001/jamacardio.2021.3471

69. Kohli U, Desai L, Chowdhury D, et al. mRNA Coronavirus-19 Vaccine-Associated Myopericarditis in Adolescents: A Survey Study. J Pediatr. 2021;S0022-3476(21)01231-2. doi:10.1016/j.jpeds.2021.12.025

70. Munro C. Covid-19: Study that claimed boys are at increased risk of myocarditis after vaccination is deeply flawed, say critics BMJ 2021; 374 :n2251 doi:10.1136/ bmj.n2251

71. Gargano JW, Wallace M, Hadler SC, Langley G, Su JR, Oster ME, Broder KR, Gee J, Weintraub E, Shimabukuro T, et al. Use of mRNA COVID-19 Vaccine After Reports of Myocarditis Among Vaccine Recipients: Update from the Advisory Committee on Immunization Practices — United States, June 2021. Morbidity Mortal Wkly Rep 2021;70:977-982. doi: 10.15585/mmwr.mm7027e2

72. Patone M, Mei XW, Handunnetthi L, Dixon S, Zaccardi F, Shankar-Hari M, et al. Risks of myocarditis, pericarditis, and cardiac arrhythmias associated with COVID-19 vaccination or SARS-CoV-2 infection. Nat Med. 2021;1-13.

73. Li M, Yuan J, Lv G, Brown J, Jiang X, Lu ZK. Myocarditis and Pericarditis following COVID-19 Vaccination: Inequalities in Age and Vaccine Types. Journal of Personalized Medicine. 2021; 
11(11):1106. https://doi.org/10.3390/ jpm11111106

74. Oster ME, Shay DK, Su JR, et al. Myocarditis Cases Reported After mRNABased COVID-19 Vaccination in the US From December 2020 to August 2021. JAMA. 2022;327(4):331-340. doi:10.1001/ jama.2021.24110

75. Prasad V, Farzaneh-Far R, Pegden W, Murthy V, Beck A. June 29, 2021. CDC's All-or-Nothing Approach to Teen COVID Vaccination Is All Wrong — The agency should revisit its latest guidance to maximize benefits and minimize risks. Disponible en: $<$ https://www. medpagetoday.com/opinion/secondopinions/93340>. Accedido 10.01.22

76. Prasad V. We need to talk about the vaccines. Public debate on side-effects is being censored. Disponible en: $<$ https:// unherd.com/2022/01/we-need-to-talkabout-the-vaccines/>. Accedido 10.01.22

77. Scary Reports of Deaths Following COVID-19 Vaccination Aren't What They Seem. MU healthcare. Disponible en: $<$ https://www.muhealth.org/our-stories/ scary-reports-deaths-following-covid19-vaccination-arent-what-they-seem $>$. Accedido 20.01.22

78. New Zealand links 26-year-old man's death to Pfizer COVID-19 vaccine. Reuters, December 20, 2021. Disponible en: <https://www.reuters.com/world/ asia-pacific/new-zealand-links-26year-old-mans-death-pfizer-covid-19vaccine-2021-12-20/>. Accedido 20.01.22

79. Malecki KMC, Keating JA, Safdar N. Crisis Communication and Public
Perception of COVID-19 Risk in the Era of Social Media. Clin Infect Dis. 2021;72(4):697-702. doi:10.1093/cid/ ciaa758

80. Lou N. Post-Vax Myocarditis Most Pervasive in High-School Boys. Disponible en: $<$ https://www. medpagetoday.com/infectiousdisease/ covid19vaccine/96844 $>$. Accedido 25.01.22

81. Zimmermann P, Pittet LF, Curtis N. Long covid in children and adolescents. BMJ. 2022;376:o143. doi:10.1136/bmj.o143

82. Zimmermann P, Pittet LF, Curtis N. How Common is Long COVID in Children and Adolescents?. Pediatr Infect Dis J. 2021;40(12):e482-e487. doi:10.1097/ INF.0000000000003328

83. Mcfarland S, Citrenbaum S, Sherwood $\mathrm{O}$, Togt $\mathrm{V}$ van der, Rossman JS. Long COVID in children. Lancet Child Adolesc Heal. 2022;6:e1. doi: 10.1016/s23524642(21)00338-2

84. Borch L, Holm M, Knudsen M, Ellermann-Eriksen S, Hagstroem S. Long COVID symptoms and duration in SARSCoV-2 positive children - a nationwide cohort study. Eur J Pediatr. 2022;1-11. doi:10.1007/s00431-021-04345-z

85. Pifarré I Arolas H, Acosta E, LópezCasasnovas G, et al. Years of life lost to COVID-19 in 81 countries [published correction appears in Sci Rep. 2021 Apr 14;11(1):8543]. Sci Rep. 2021;11(1):3504. doi:10.1038/s41598-021-83040-3

86. Mahajan A, Phuong L, Nguyen T, et al. 50 Global Actions to Manage the COVID-19 Infodemic: A WHO Framework European
Journal of Public Health. 2021;31(Suppl 3). doi:10.1093/eurpub/ckab164.277

87. Rodriguez-Morales AJ, Franco OH. Public trust, misinformation and COVID-19 vaccination willingness in Latin America and the Caribbean: today's key challenges. Lancet Regional Heal - Am 2021;3:100073. doi: 10.1016/j. lana.2021.100073

88. Donovan J. Recomendaciones concretas para contrarrestar la información errónea durante la pandemia de COVID-19. Revista Panam De Salud Pública. 2021;45:e59.

89. Limaye RJ, Sauer M, Ali J, Bernstein J, Wahl B, Barnhill A, Labrique A. Building trust while influencing online COVID-19 content in the social media world. Lancet Digital Heal (2020) 2:e277-e278. doi: 10.1016/s2589-7500(20)30084-4

90. Lewnard JA, Lo NC. Scientific and ethical basis for social-distancing interventions against COVID-19. Lancet Infect Dis. 2020;20(6):631-633. doi:10.1016/S14733099(20)30190-0

91. González-Dambrauskas S. Coronnials y cuarenteens. La diaria, opinión, 29.06.20. Disponible en: <https://ladiaria.com.uy/ opinion/articulo/2020/6/coronnials-ycuarenteens/> Accedido 22.01.22

92. HaleT, Angrist N, Goldszmidt R, et al. "A global panel database of pandemic policies (Oxford COVID-19 Government Response Tracker).” Nature Human Behaviour. 2021. doi: 10.1038/s41562021-01079-8. Disponible en: < https:// github.com/OxCGRT/covid-policytracker > Accedido 27.01.22. 